\title{
Factors associated with safe early discharge after transcatheter aortic valve implantation
}

\author{
Omar Aldalati, Friedon Keshavarzi, Amit Kaura, Jonathan Byrne, Mehdi Eskandari,
} Ranjit Deshpande, Mark Monaghan, Olaf Wendler, Rafal Dworakowski, Philip MacCarthy

King's College Hospital, Denmark Hill, London, United Kingdom

\begin{abstract}
Background: As transcatheter aortic valve implantation (TAVI) becomes more straightforward, a larger proportion of patients will be well enough to be discharged early. This study sought to characterise the clinical features that allowed patients to be discharged early after TAVI and to evaluate the safety of an early discharge policy.

Methods: All patients undergoing TAVI at the above cited center from August 2007 to March 2015 were included in this study. Baseline characteristics, in-hospital outcomes, re-admissions and mortality were compared.

Results: Three hundred thirty-seven TAVIs were performed during the study period, and 18 died in-hospital (18/337, 5.3\%). Of the remaining patients, 56 were discharged within 3 days of the index procedure ('early discharge group' 56/319, 17.5\%). There was no difference between the early discharge and late discharge group in terms of Valve Academic Research Consortium-2 (VARC-2) criteria outcomes, all-cause re-admission rates and the need for permanent pacemaker implantation. Mortality at 1 year was better among the early discharge group (3.6\% vs. 15.6\%, $p=0.014)$; a reflection of baseline clinical differences.

Conclusion: Early discharge of clinically selected TAVI patients is safe and appropriate. Lower logistic EuroSCORE, smaller delta creatinine and not developing any complications are factors associated with early discharge. (Cardiol J 2018; 25, 1: 14-23)

Key words: transcatheter aortic valve implantation, transcatheter aortic valve replacement, early discharge, Valve Academic Research Consortium-2 (VARC-2), safety, re-admissions
\end{abstract}

\section{Introduction}

Transcatheter aortic valve implantation (TAVI) has become the 'standard of care' for the treatment of high risk and surgically inoperable patients with symptomatic aortic stenosis (AS). The PARTNER 2 [1] trial has also recently demonstrated that TAVI is non-inferior to surgical aortic valve replacement (sAVR) in the 'intermediate risk' group. The number of TAVIs performed world-wide is therefore likely to increase significantly in the near future. Moreover, recent years have seen impressive reductions in complications of TAVI and consequent improvements in outcomes and survival, which was evident in PARTNER 2. TAVI is becoming a more straightforward and reproducible procedure and the move towards conscious sedation, smaller delivery systems and improvements in percutaneous closure techniques have allowed TAVI patients to mobilize soon after the procedure, facilitating early discharge. Early discharge of TAVI patients will improve cost-effectiveness; data already indicate that despite greater procedural costs, TAVI is cost-effective compared with sAVR over a 10 -year period, due to the greater post-procedural cost of sAVR [2]. In order to respond to an increasing

Address for correspondence: Rafal Dworakowski, MD, PhD, Department of Cardiology, King's College Hospital, Denmark Hill, London, SE5 9RS, United Kingdom, tel: ++44-20-3299-3379, e-mail: rdworakowski@nhs.net

Received: 28.03.2017

Accepted: 11.07.2017 
demand for TAVI and to improve the efficiency of our patient pathway, this research sought to study the factors associated with safe early discharge in a "real world" population after the TAVI procedure.

\section{Methods}

All cases analyzed were done between August 2007 and March 2015. Each patient was selected for TAVI by a multidisciplinary Heart Team that consisted of specialist nurses, interventional and imaging Cardiologists and Cardiothoracic Surgeons. Patients underwent pre-TAVI work up including trans-oesophageal echocardiography, coronary angiography and peripheral angiography as well as computed tomography (CT) aortography. The aortic valve annulus was sized using three-dimensional (3D) trans-oesophageal echocardiography. This was the first centre in the United Kingdom to implant an Edwards Sapien valve (Edwards Lifesciences, Irvine, California) and, as such, it is the predominantly used prosthesis herein. During the study period, general anaesthetics had been the default method of anaesthesia and only 2 cases were performed under conscious sedation.

From 2007 to 2012 , patients were routinely monitored on the Intensive Care Unit post-TAVI. Since October 2012, patients have been cared for on a level-two unit post-TAVI such as Coronary Care Unit/High Dependency Unit. Presented protocol mandates a minimum $24 \mathrm{~h}$ stay in a level two ward followed by a step-down to a cardiology/ /cardiothoracic ward (level one) with early mobilisation and physiotherapy. On day one or two (day of index procedure is day 0 ), patients undergo transthoracic echocardiography. If clinically stable, the majority of patients will be considered for planned discharge on day two.

Patient demographics, detailed procedural characteristics, hospital stay and outcomes are recorded prospectively on a national database and reported according to Valve Academic Research Consortium-2 (VARC-2) criteria [3]. Mortality and re-admission rates were obtained from local and national databases. Based on length of hospital stay, cases were classified into early discharge ( $\leq 3$ days) and late discharge ( $>3$ days). In order to devise an early discharge protocol, a "safe outcome" measure was created:

- "Safe outcome at 30 days" was defined as absence of: 1) pacing post discharge up to 30 days, 2) any cause re-admission up to 30 days, and 3 ) any cause mortality up to 30 days.
— "Safe outcome at 1 year" was defined as $a b$ sence of: 1) pacing post discharge up to 1 year, 2) any cause re-admission up to 1 year, and 3 ) any cause mortality up to 1 year.

\section{Statistical analysis}

Continuous variables are expressed as means and standard deviations. Parametric and nonparametric tests were used based on sample size and normality of distribution. Categorical data are expressed as percentages. Univariable analysis using $\chi^{2}$, Fisher exact, or $\mathrm{t}$ test, ANOVA and other non-parametric tests were used to identify differences between early and late discharge groups.

Multiple imputations were used to replace missing data when the variable had less than $10 \%$ missing values. "Automatic Imputation Method" was used with five imputations. Pooled data were analyzed.

In order to determine the factors associated with early discharge, logistic regression model was generated. Seven variables were included in the model to permit for the rule of thumb of " $5-9$ events per variable" [4]. The variables were chosen based on univariate analysis and what has already been published. The same model was then used to identify predictors of "safe outcome at 1 year".

All analysis was done using SPSS statistical software (IBM Corp. Released 2013. IBM SPSS Statistics for Windows, Version 22.0. Armonk, NY: IBM Corp).

\section{Results}

From August 2007 until March 2015, 337 TAVI procedures were performed in the above cited center. Eighteen $(5.4 \%)$ patients died in hospital. Fifty-six $(56 / 319 ; 17.5 \%)$ patients were discharged home early ( $\leq 3$ days post-procedure). Figure 1 shows the number of days spent in hospital. The baseline characteristics of the study group are summarized in Table 1. Just over half of our patients were male $(50.2 \%)$. The mean age was $82.58 \pm 6.7$ years. The mean hospital stay was $9.4 \pm 9.7$ days (mode 3 days, median 6 days). The median hospital stay among the early discharge group was 3 (interquartile range [IQR]: $3-3$ ) days and among late discharge group 7 (IQR: $5-13$ ) days, $\mathrm{p}<0.0001$.

During the study period, the mean sheath size dropped significantly from $24 \mathrm{~mm}$ to $17 \mathrm{~mm}$ $(p<0.0001)$ and the percentage of trans-apical cases performed decreased from $51 \%$ to $17.4 \%$ ( $\mathrm{p}<0.0001)$. Moreover, the mean logistic EuroSCORE also dropped (22 to $15, \mathrm{p}<0.0001$ ). The 


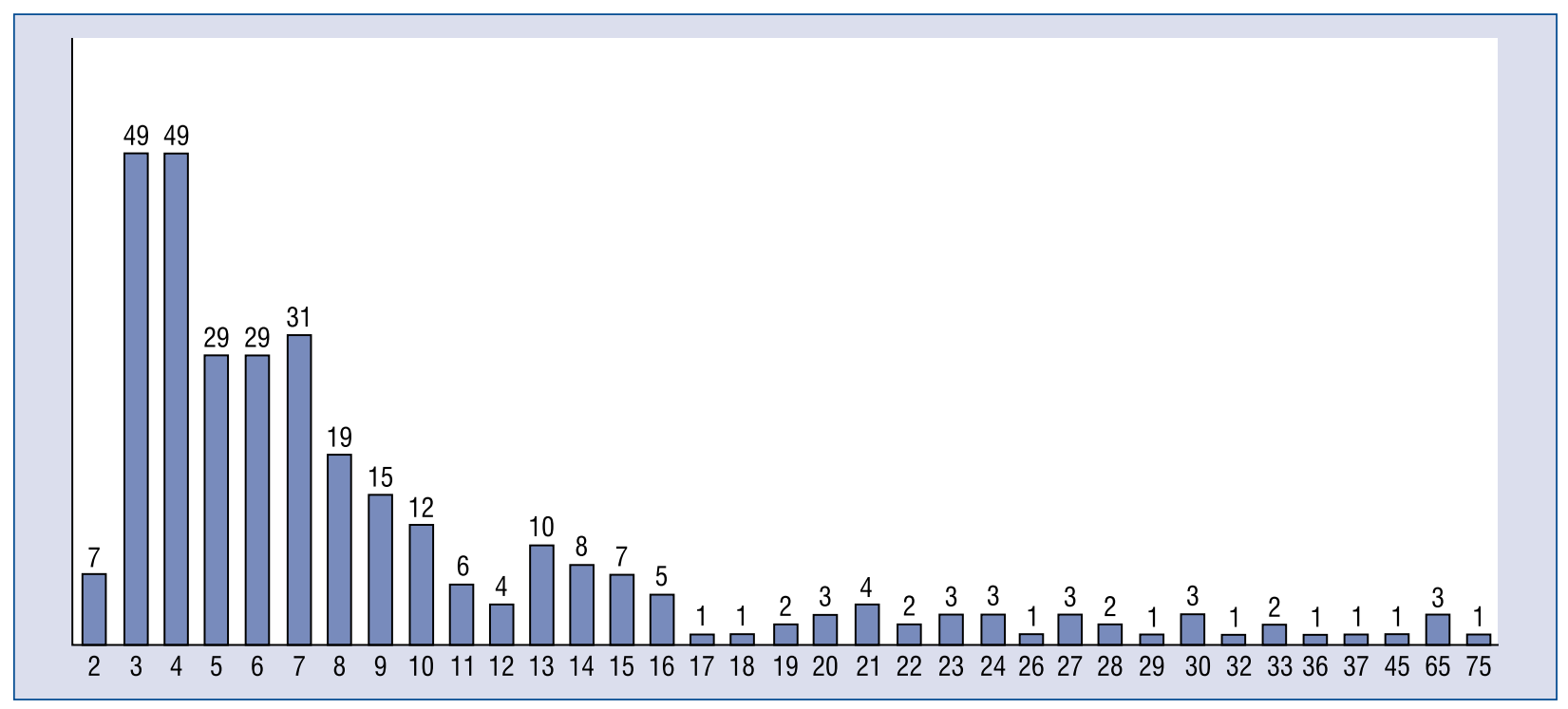

Figure 1. Hospital stay in days; Legend: frequency of hospital stay based on number of days.

above changes were associated with a significant rise in the percentage of early discharge (2\% to $32 \%, \mathrm{p}<0.0001)$ and a reduction in the overall mean hospital stay (11.4 to 7.1 days, $\mathrm{p}<0.0001$ ). Only two cases were done under conscious sedation, both were performed in March 2015 and were discharged early.

Patients discharged early had a lower New York Heart Association (NYHA) class III/IV (48\% vs. $66 \%, p=0.001)$, lower rates of peripheral vascular disease, lower baseline creatinine and a lower mean logistic EuroSCORE (16.7 vs. 21.7, $\mathrm{p}=0.001)$ (Table 1). Echocardiographic features showed less aortic valve (AV) calcification and a larger AV area in the early discharge group (Table 1).

The procedural characteristics were also different between the two groups. Among patients discharged early, the trans-apical route was used less, the sheath size was smaller, the duration of rapid pacing was shorter periprocedurally, and percutaneous closure devices were more frequently used (Table 2). Patients discharged early had a shorter level-two stay (0.93 vs. 1.43 days, $\mathrm{p}<0.0001$ ).

Adjudicated according to VARC-2 criteria, life-threatening bleeding and acute kidney injury (AKI) stage II or III were higher among late discharge group at 30 days. There were no deaths among the early discharge group within 30 days of discharge and $3(1.1 \%)$ patients died from the late discharge group $(\mathrm{p}=1)$. Within 1 year, $2(3.6 \%)$ and 41 (15.6\%) patients died, respectively $(\mathrm{p}=0.014)$; Table 3 summaries outcomes according to VARC-2 criteria.
Moreover, patients in the early discharge group required less blood transfusion, and had lower delta creatinine (the difference between the highest creatinine level after the procedure and the level before) and a lower hemoglobin drop post-TAVI, a potential surrogate marker for bleeding (Table 3).

Using multivariable logistic regression, the following factors were associated with early discharge: logistic EuroSCORE (odds ratio [OR] $0.947,95 \%$ confidence interval [CI] 0.912-0.983, $\mathrm{p}=0.004)$, delta creatinine (OR $0.967,95 \% \mathrm{CI}$ 0.949-0.985, $\mathrm{p}<0.0001$ ) and development of any complication as defined by an occurrence of any of the following - stroke, major or life threatening bleeding, major vascular injury, AKI stage II/III or the need for pacing (OR 0.174, 95\% CI 0.048-0.630, $\mathrm{p}=0.008$ ). Factors such as prior pacing, NYHA class and bleeding did not influence early discharge in this heterogenous group of patients when accounting for other variables. Table 4 summarizes univariate and multivariate predictors of early discharge.

All-cause re-admission rate and the need for permanent pacing after discharge were also studied. In the early discharge group, only 2 patients required re-admission within 30 days $(2 / 56,3.6 \%)$ whilst there were 25 re-admissions among the late discharge group $(25 / 263,9.5 \%, \mathrm{p}=0.19)$. The early discharge group had 9 (16.1\%) admissions between 30 days and 12 months after discharge whilst the late discharge group of patients had $56(21.3 \%)$ admissions during the same period $(\mathrm{p}=0.4)$. $64 \%$ and $54 \%$ of these re-admissions were car- 
Table 1. Baseline characteristics.

\begin{tabular}{|c|c|c|c|c|}
\hline Variable & Overall & $\begin{array}{l}\text { Early discharge } \\
N=56(17.5 \%)\end{array}$ & $\begin{array}{c}\text { Late discharge } \\
N=263(82.5 \%)\end{array}$ & $\mathbf{P}$ \\
\hline Logistic EuroSCORE [\%] & $20.8(10)$ & $16.7(9)$ & $21.7(11)$ & 0.0001 \\
\hline Male & $160(50.2 \%)$ & $27(48 \%)$ & $133(49 \%)$ & 0.4 \\
\hline Age [years] & $82.58(6.7)$ & $81.8(7.7)$ & $82.75(6.5)$ & 0.6 \\
\hline \multicolumn{5}{|l|}{ Indication for TAVI: } \\
\hline High risk & $203(63.6 \%)$ & $26(46 \%)$ & $177(67 \%)$ & 0.009 \\
\hline Surgical turn down & $116(36.4 \%)$ & $30(54 \%)$ & $86(33 \%)$ & \\
\hline CCS angina class III or IV & $30(9.4 \%)$ & $2(3.5 \%)$ & $28(10.6 \%)$ & 0.2 \\
\hline NYHA class III or IV & $201(63 \%)$ & $27(48 \%)$ & $174(66 \%)$ & 0.001 \\
\hline Diabetes mellitus & $60(18.8 \%)$ & $10(17.8 \%)$ & $50(19 \%)$ & 0.4 \\
\hline Smoking & $13(4.1 \%)$ & 0 & $13(4.9 \%)$ & 0.02 \\
\hline Hypertension & $250(78.4 \%)$ & $43(77 \%)$ & $207(79 \%)$ & 0.3 \\
\hline Hyperlipidemia & $180(56.4 \%)$ & $29(52 \%)$ & $151(57 \%)$ & 0.4 \\
\hline Creatinine $[\mu \mathrm{mol} / \mathrm{L}]$ & $108(86)$ & $93.8(36)$ & $111(93)$ & 0.021 \\
\hline eGFR $[\mathrm{mL} / \mathrm{min}]$ & $60(20)$ & $65.6(19)$ & $59(20)$ & 0.028 \\
\hline Previous myocardial infarction & $23(7.2 \%)$ & $7(12.5 \%)$ & $16(6 \%)$ & 0.08 \\
\hline Pulmonary disease & $90(28.2 \%)$ & $14(5.3 \%)$ & $76(29 \%)$ & 0.2 \\
\hline Neurological disease & $46(14.4 \%)$ & $6(11 \%)$ & $40(15 \%)$ & 0.2 \\
\hline Carotid disease $>50 \%$ stenosis & $63(19.7 \%)$ & $10(18 \%)$ & $53(20 \%)$ & 0.4 \\
\hline Peripheral vascular disease & $78(24.5 \%)$ & $8(14 \%)$ & $70(27 \%)$ & 0.034 \\
\hline Extra cardiac arteriopathy & $115(36.1 \%)$ & $15(27 \%)$ & $100(38 \%)$ & 0.07 \\
\hline Sinus rhythm & $180(56.4 \%)$ & $31(55 \%)$ & $149(57 \%)$ & 0.6 \\
\hline Previous cardiac surgery & $80(25.1 \%)$ & $12(21 \%)$ & $68(26 \%)$ & 0.3 \\
\hline Previous $\mathrm{PCl}$ & $52(16.3 \%)$ & $7(12.5 \%)$ & $45(17 \%)$ & 0.2 \\
\hline Two or three vessel CAD & $53(17.7 \%)$ & $8(14 \%)$ & $45(17 \%)$ & 0.3 \\
\hline Left main stem disease & $10(3.1 \%)$ & $1(2 \%)$ & $9(3.5 \%)$ & 0.6 \\
\hline FEV1 [\%] & $81.25(27)$ & $82.32(21)$ & $80.7(28)$ & 0.6 \\
\hline Elective procedure & $311(97.4 \%)$ & $56(100 \%)$ & $255(97 \%)$ & 0.1 \\
\hline QRS duration [ms] & $114(34)$ & $123(39)$ & $112(32)$ & 0.057 \\
\hline Poor left ventricular function* & $12(3.8 \%)$ & $4(7 \%)$ & $8(3 \%)$ & 0.4 \\
\hline Mixed aortic valve pathology & $4(1.3 \%)$ & $2(3.5 \%)$ & $2(0.8 \%)$ & 0.3 \\
\hline Degenerative aortic valve etiology & $297(93.1 \%)$ & $52(93 \%)$ & $245(93 \%)$ & 0.9 \\
\hline Extensive calcification of aortic valve & $88(27.6 \%)$ & $7(12.5 \%)$ & $81(31 \%)$ & 0.003 \\
\hline Mean aortic gradient [mm Hg] & $44(15)$ & $40(14)$ & $44(14)$ & 0.8 \\
\hline Peak aortic gradient [mm Hg] & $77(24$ & $72(25)$ & $78(23)$ & 0.1 \\
\hline Aortic valve area $\left[\mathrm{cm}^{2}\right]$ & $0.82(0.4)$ & $0.93(0.3)$ & $0.8(0.4)$ & 0.008 \\
\hline
\end{tabular}

*Ejection fraction < 30\%; CAD - coronary artery disease; CCS - Canadian Cardiovascular Society; eGFR - estimated glomerular filtration rate; FEV1 - forced expiratory volume in the first second; NYHA - New York Heart Association; PCl - percutaneous coronary intervention

diovascular in origin at 30 days and 12 months, respectively (causes of these admissions are shown in Table 5). Up to 30-days post discharge, 2 (3.6\%) patients out of 56 needed permanent pacing in the early discharge group but only $1(0.4 \%)$ patient in the late discharge group. By 12 months, 2 (3.6\%) more patients required permanent pacing in the early discharge group and $4(1.5 \%)$ in the late discharge group $(\mathrm{p}=0.56)$.

"Safe outcome at 30 days and 1 year" was reached in $287(90 \%)$ and $213(67 \%)$ cases respectively in the overall cohort. At 30 days, $93 \%$ of early discharge patients had safe outcomes vs. $89 \%$ in the late discharge group $(\mathrm{p}=0.62)$, and 
Table 2. Procedural characteristic.

\begin{tabular}{|c|c|c|c|c|}
\hline Variable & Overall & Early discharge & Late discharge & $\mathbf{P}$ \\
\hline Procedure time [min] & $106.64(66.9)$ & $101(121)$ & $108(42)$ & 0.0001 \\
\hline Conscious sedation & $2(0.6 \%)$ & $2(3.5 \%)$ & 0 & 0.12 \\
\hline Delivery approach: & & & & 0.0001 \\
\hline TF-PC & $111(34.8 \%)$ & $40(71 \%)$ & $71(27 \%)$ & \\
\hline TF-surgical cut down & $90(28.2 \%)$ & $12(22 \%)$ & $78(30 \%)$ & \\
\hline TA & $115(36.1 \%)$ & $4(7 \%)$ & $111(42 \%)$ & \\
\hline Other & $3(0.9 \%)$ & 0 & $3(1 \%)$ & \\
\hline Sheath size [Fr] & $19.97(4)$ & $17.4(3.2)$ & $20.5(3.9)$ & 0.0001 \\
\hline Valve type: & & & & 0.0001 \\
\hline Sapien XT & $248(77.7 \%)$ & $31(55 \%)$ & $217(82 \%)$ & \\
\hline Sapien 3 & $60(18.8 \%)$ & $20(36 \%)$ & $40(16 \%)$ & \\
\hline Others & $11(3.4 \%)$ & $5(9 \%)$ & $6(2 \%)$ & \\
\hline Valve size $[\mathrm{mm}]$ & $25.1(2)$ & $25(1.9)$ & $25(2)$ & 0.9 \\
\hline Pacing time $[\mathrm{s}]$ & $50(37)$ & $36(24)$ & $53(39)$ & 0.0001 \\
\hline Volume of contrast [mL] & $116(53)$ & $116(52)$ & $116(54)$ & 0.9 \\
\hline Vascular closure technique: & & & & 0.0001 \\
\hline Percutaneously & $114(35.7 \%)$ & $41(73 \%)$ & $73(28 \%)$ & \\
\hline Surgical & $205(64.3 \%)$ & $15(27 \%)$ & $190(72 \%)$ & \\
\hline
\end{tabular}

CVA - cerebrovascular accident; TA - trans-apical; TF-PC — trans-femoral percutaneous

at 1 year, $79 \%$ vs. $64 \%$, respectively $(\mathrm{p}=0.026)$. Likely, a reflection of baseline clinical differences between the two groups i.e. patients who were discharged early were healthier. At 1 year, logistic EuroSCORE (OR 0.973, 95\% CI 0.951-0.996, $\mathrm{p}=0.020)$, delta creatinine (OR $0.996,95 \% \mathrm{CI}$ $0.992-1.000, \mathrm{p}=0.048$ ) and prior pacing (OR $2.808,95 \%$ CI $1.122-7.011, \mathrm{p}=0.027$ ) were the predictors of a safe outcome.

\section{Discussion}

Despite high risk characteristics of this patient group, the present data and the recently published works by Durand et el. [5] and Barbanti et al. [6] suggest that early discharge ( $\leq 3$ days) is a feasible and safe option after TAVI. Factors associated with early discharge seem to be consistent in these studies and they overlap with predictors of "safe outcome". Combining these factors/predictors, it was possible to create a protocol to facilitate early discharge (Fig. 2).

One of the major concerns post-TAVI is the risk of potentially dangerous conduction abnormalities. According to Khawaja et al. [7], the overall median time to permanent pacemaker implantation post TAVI was 4 days. However, this study looked at CoreValve prostheses (Medtronic, Minneapolis,
Minnesota, USA) the use of which is associated with higher rates of pacing than following Edwards Sapien implantation. Another study utilizing both self-expanding and balloon-expandable TAVI valves has shown that pacemaker requirement becomes apparent at 4 days [8]. However, neither studies report on the time of diagnosis of conduction defects post TAVI. This study indicates that early discharge does not increase the risk of re-admission for pacemaker implantation. Presumably, this suggests that conduction defects become evident early post TAVI i.e. in the first 3 days.

The negative impact of bleeding and blood product transfusion on hospital stay with a variety of cardiac procedures is well known and its influence on the TAVI patient cohort is also recognized. Barbanti et al. [6] demonstrated that lack of significant bleeding is a predictor of early discharge whilst Durand et al. [5] demonstrated that requirement of blood transfusion post-TAVI was a negative predictor of early discharge. The present group of patients was more heterogenous than those included in these studies and, as such, neither bleeding nor transfusion feature as significant predictors of early discharge on multivariate analysis. Nonetheless, improving procedural techniques to reduce bleeding and blood transfusion are likely to improve rate of early discharge. 
Table 3. Post-procedural outcomes.

\begin{tabular}{|c|c|c|c|c|}
\hline Variable & $\begin{array}{l}\text { Overall } \\
(n=319)\end{array}$ & $\begin{array}{l}\text { Early discharge } \\
\qquad(\mathrm{n}=56)\end{array}$ & $\begin{array}{l}\text { Late discharge } \\
\qquad(\mathrm{n}=263)\end{array}$ & $\mathbf{P}$ \\
\hline \multicolumn{5}{|l|}{ Early safety (at 30 days) } \\
\hline All-cause mortality & $3(99.1 \%)$ & 0 & $3(1.1 \%)$ & 0.55 \\
\hline All stroke (includes 4 TIAs) & $10(3 \%)$ & 0 & $10(3.8 \%)$ & 0.21 \\
\hline Life threatening bleeding & $21(6.5 \%)$ & 0 & $21(8 \%)$ & 0.03 \\
\hline AKI stage 2 or 3 & $24(7.5 \%)$ & 0 & $24(9 \%)$ & 0.02 \\
\hline Coronary obstruction & 0 & 0 & 0 & N/A \\
\hline Major vascular complications & $15(4.7 \%)$ & 0 & $15(5.7 \%)$ & 0.08 \\
\hline Lack of intended performance of prosthestheic heart valve & $24(7.5 \%)$ & $8(14 \%)$ & $16(6 \%)$ & 0.04 \\
\hline \multicolumn{5}{|l|}{ Clinical efficacy (after 30 days) } \\
\hline All-cause mortality & $43(13.5 \%)$ & $2(3.6 \%)$ & $41(15.6 \%)$ & 0.014 \\
\hline All stroke & $3(1 \%)$ & $1(1.7 \%)$ & $2(0.75 \%)$ & 0.99 \\
\hline Hospitalization for valve-related symptoms or heart failure & $8(2.5 \%)$ & $2(3.6 \%)$ & $6(2.2 \%)$ & 0.63 \\
\hline NYHA III or IV & $24(7.5 \%)$ & $2(3.6 \%)$ & $22(8.3 \%)$ & 0.27 \\
\hline Valve-related dysfunction & $45(14 \%)$ & $6(10.7 \%)$ & $39(14.8 \%)$ & 0.52 \\
\hline \multicolumn{5}{|l|}{ Time-related valve safety ( 30 days up to a 1 year) } \\
\hline Valve related dysfunction & $45(14 \%)$ & $6(10.7 \%)$ & $39(14.8 \%)$ & 0.52 \\
\hline Valve endocarditis & $3(1 \%)$ & $1(1.7 \%)$ & $2(0.8 \%)$ & 0.99 \\
\hline Valve thrombosis & 0 & 0 & 0 & N/A \\
\hline Thrombo-embolic events* & $3(1 \%)$ & $1(1.7 \%)$ & $2(0.75 \%)$ & 0.99 \\
\hline VARC bleeding $\$$ & 0 & 0 & 0 & N/A \\
\hline \multicolumn{5}{|l|}{ Outcomes that are not captured by VARC-2 } \\
\hline Level two stay [day] & $1.34(1.6)$ & $0.93(0.68)$ & $1.43(1.8)$ & 0.0001 \\
\hline Delta creatinine & $27(72)$ & $-3(13)$ & $33(77)$ & 0.0001 \\
\hline Haemoglobin drop & $26(16)$ & $21(11)$ & $27(17)$ & 0.0001 \\
\hline Blood transfusion & $80(25 \%)$ & $3(5 \%)$ & $77(29 \%)$ & 0.0001 \\
\hline GI bleed & $2(0.6 \%)$ & 0 & $2(0.6 \%)$ & 1 \\
\hline
\end{tabular}

*Based on stroke events. \$ There were three cases of upper gastrointestinal bleeds that were not related to transcatheter aortic valve replacement (TAVI); GI — gastro-intestinal, creatinine (umol/L), hemoglobin (g/L); AKI — acute kidney injury; N/A — not applicable; NYHA - New York Heart Association; VARC-2 - Valve Academic Research Consortium-2; TIA — transient ischemic attack

The predictive value associated with serum creatinine seems consistent in this study and others $[5,6]$. AKI post-TAVI is associated with increased mortality [9] but studies on outcome post-TAVI and chronic kidney disease have shown inconsistent results $[10,11]$ thus far. The influence of these conditions on hospital stay has not been studied in detail. The reported OR on post-TAVI delta creatinine demonstrates clearly that AKI has a negative impact on early discharge rates.

The occurrence of any complication influence discharge. It is noteworthy that Durand et al. [5] showed that most of the major complications post TAVI occurred within $48 \mathrm{~h}$ after the procedure i.e. patients who developed complications were known by day three and therefore not discharged. The study also reports that only 3 patients had delayed major complications.

Based on current evidence, factors associated with early discharge are therefore prior pacemaker (OR 2.27-2.8), and a more recently performed procedure (a reflection of the advancement of the technique, OR 1.66), NYHA IV (OR 0.22), logistic EuroSCORE (OR 0.94), bleeding (OR 0.31), transfusion (OR 0.10), previous balloon aortic valvuloplasty (OR 0.44), delta creatinine (OR 0.96-0.99), and developing a complication (OR 0.17).

This study demonstrates that clinically selected early discharge is associated with similar allcause re-admission rate, similar 30-day mortality and similar re-admission rate for permanent pacing. These findings are consistent with other published 
Table 4. Univariate and multivariate predictors of early discharge.

\begin{tabular}{|c|c|c|c|}
\hline Variable & Odds ratio & $95 \% \mathrm{Cl}$ & $\mathbf{P}$ \\
\hline \multicolumn{4}{|l|}{ Univariate analysis } \\
\hline Sex & 1.08 & $0.608-1.908$ & 0.788 \\
\hline Age & 0.992 & $0.960-1.024$ & 0.619 \\
\hline Body mass index & 1.024 & $0.995-1.055$ & 0.110 \\
\hline Logistic EuroSCORE & 0.949 & $0.918-0.981$ & 0.002 \\
\hline Diabetes mellitus & 0.926 & $0.437-1.961$ & 0.841 \\
\hline Hypertension & 0.895 & $0.450-1.779$ & 0.751 \\
\hline Creatinine pre-TAVR & 0.995 & $0.987-0.1002$ & 0.166 \\
\hline NYHA III/IV & 0.476 & $0.266-0.853$ & 0.013 \\
\hline Previous MI & 2.205 & $0.862-5.643$ & 0.099 \\
\hline PVD & 2.176 & $0.981-4.828$ & 0.056 \\
\hline$E F<30 \%$ & 2.490 & $0.723-8.582$ & 0.148 \\
\hline Paced pre-TAVR & 0.622 & $0.276-1.404$ & 0.253 \\
\hline AV mean gradient & 0.980 & $0.958-1.001$ & 0.065 \\
\hline Procedure time & 0.998 & $0.991-1.004$ & 0.525 \\
\hline Delta creatinine & 0.973 & $0.959-0.987$ & 0.001 \\
\hline Hemoglobin drop $[\mathrm{g} / \mathrm{L}]$ & 0.975 & $0.957-0.994$ & 0.009 \\
\hline Blood transfusion & 0.137 & $0.041-0.451$ & 0.001 \\
\hline Life-threatening bleed & 0.233 & $0.031-1.781$ & 0.161 \\
\hline Any complication & 0.141 & $0.049-0.401$ & 0.001 \\
\hline \multicolumn{4}{|l|}{ Multivariate analysis } \\
\hline Logistic EuroSCORE & 0.947 & $0.912-0.983$ & 0.004 \\
\hline NYHA III/IV & 0.748 & $0.375-1.491$ & 0.410 \\
\hline Paced pre-TAVI & 0.967 & $0.645-4.338$ & 0.290 \\
\hline Delta creatinine & 0.967 & $0.949-0.985$ & 0.001 \\
\hline Any complication & 0.174 & $0.048-0.630$ & 0.008 \\
\hline Life-threatening bleed & 1.777 & $0.151-20.967$ & 0.648 \\
\hline Blood transfusion & 0.429 & $0.119-1.554$ & 0.198 \\
\hline
\end{tabular}

AV - aortic valve; $\mathrm{Cl}$ - confidence interval; EF — ejection fraction; $\mathrm{MI}$ - myocardial infarction; NYHA — New York Heart Association; PVD — peripheral vascular disease; TAVI — trancatheter aortic valve implantation

studies [5, 6]. It also shows that early discharge is associated with a safe outcome for up to 1 year. The current trend in practice towards conscious sedation may influence the early discharge rate further; nonetheless, the present experience shows safe early discharge even for those performed under general anaesthesia. Overlap between factors associated with early discharge and predictors of safe outcome provides us with an opportunity to devise a clinical protocol that incorporates these predictors (Fig. 2).

Safe early discharge is likely to significantly improve cost-effectiveness of TAVI. Babaliaros et al. [12], as demonstrated previously, that with a 'minimalist approach' with TF TAVI, shorter length of stay translated into lower resource use and significantly lower hospital costs. The same is likely to apply to early discharge.

This is a retrospective observational study with the inherent limitations of any observational study. Physical performance status and family support are significant confounders of early discharge among the elderly TAVI patients. Assessment of frailty and quality of life was introduced recently into the TAVI practice and as such quantifying these important social issues in retrospect is difficult and was not included in the analysis.

All patients included who received this technology throughout its evolution and practice has changed significantly. It is well known that the TAVI procedure in the current era is significantly different from 2007 and as such it is appreciated 
Table 5. List of causes of re-admissions.

\begin{tabular}{|c|c|c|c|}
\hline \multicolumn{2}{|c|}{ 30-day re-admissions } & \multicolumn{2}{|c|}{ 12-month re-admissions } \\
\hline Cardiovascular & Non-cardiovascular & Cardiovascular & Non-cardiovascular \\
\hline \multirow{5}{*}{$\begin{array}{l}\text { Bilateral groin hematomas } \\
\text { needing IV antibiotics } \\
\text { Right femoral pseudo- } \\
\text {-aneurysm needing thrombin } \\
\text { Right femoral pseudo- } \\
\text {-aneurysm }\end{array}$} & LRTI (2 cases) & \multirow{2}{*}{$\begin{array}{l}\text { Syncope with bi-fascicular } \\
\text { block needing a PPM }\end{array}$} & Falls ( 2 cases) \\
\hline & & & \multirow{2}{*}{$\begin{array}{l}\text { Urology admission for } \\
\text { flexible cystoscopy ( } 2 \text { cases }\end{array}$} \\
\hline & 'Not coping at home' & $\begin{array}{l}\text { Non cardiac chest pain } \\
\text { ( } 2 \text { cases) }\end{array}$ & \\
\hline & Pneumonia with pleural & Repair of femoral pseudo- & Pelvic tracture \\
\hline & $\begin{array}{l}\text { effusion needing a chest } \\
\text { drain }\end{array}$ & $\begin{array}{l}\text { aneurysm (TAVI complica- } \\
\text { tion, } 2 \text { cases) }\end{array}$ & $\begin{array}{l}\text { Readmission for OGD. } \\
\text { Had UGI bleed post TAVI }\end{array}$ \\
\hline \multirow{3}{*}{$\begin{array}{l}\text { Left pleural effusion } \\
\text { (conservative management) } \\
\text { Left pleural effusion requiring } \\
\text { a chest drain }\end{array}$} & Sepsis & \multirow{3}{*}{$\begin{array}{l}\text { Elective admission for } \\
\text { right femoral AVF } \\
\text { exploration and wound } \\
\text { debridement (TAVI } \\
\text { complication) }\end{array}$} & Elective CEA \\
\hline & & & Ophthalmology (2 cases) \\
\hline & & & Hyperkalaemia secondary \\
\hline \multicolumn{2}{|l|}{ Groin Seroma } & \multirow{2}{*}{$\begin{array}{l}\text { Post-operative external } \\
\text { apical clot (echocardio- } \\
\text { graphy surveillance) }\end{array}$} & ineffective dialysis line \\
\hline $\begin{array}{l}\text { Fuid overload needing } \\
\text { IV diuretics ( } 2 \text { cases) }\end{array}$ & & & Confusion \\
\hline \multicolumn{2}{|l|}{ Groin swelling ( 3 cases) } & \multirow{2}{*}{$\begin{array}{l}\text { Infected groin wound } \\
\text { needing IV antibiotics }\end{array}$} & Syncope due to recurrent \\
\hline Fast $\mathrm{AF}$ and $\mathrm{CCF}$ & & & postural hypotension \\
\hline \multirow[t]{26}{*}{ Elective admission for EVAR } & & \multirow{2}{*}{$\begin{array}{l}\text { Exercise induced cerebral } \\
\text { hypo-perfusion due to } \\
\text { bilateral carotid artery } \\
\text { stenosis }\end{array}$} & $\begin{array}{l}\text { Dyspnea due to idiopathic } \\
\text { anemia }\end{array}$ \\
\hline & & & Non-cardiac dyspnea \\
\hline & & $\begin{array}{l}\text { Investigation for stroke } \\
\text { (no evidence of stroke) }\end{array}$ & $\begin{array}{l}\text { Acute confusion needing } \\
\text { intubation }\end{array}$ \\
\hline & & CRT upgrade & UGI bleed (3 cases) \\
\hline & & $\begin{array}{l}\text { Dyspnea due to CCF } \\
\text { (4 cases) }\end{array}$ & $\begin{array}{l}\text { Closed loop bowel } \\
\text { obstruction in a back- }\end{array}$ \\
\hline & & $\begin{array}{l}\text { TAVI groin wound } \\
\text { infection }\end{array}$ & Fall \\
\hline & & \multirow{2}{*}{$\begin{array}{l}\text { Chest pain at rest needing } \\
\text { LAD-PCI ( } 2 \text { cases) }\end{array}$} & Septic shock with MOF \\
\hline & & & Pulmonary embolism \\
\hline & & \multirow{2}{*}{$\begin{array}{l}\text { Investigation for IE (no } \\
\text { evidence of IE) ( } 2 \text { cases) }\end{array}$} & ( 2 cases) \\
\hline & & & Hematology admission \\
\hline & & \multirow{4}{*}{$\begin{array}{l}\text { Worsening dyspnea due to } \\
\text { pulmonary hypertension } \\
\text { TIA } \\
\text { IE of TAVI ( } 2 \text { cases) } \\
\text { Atrial tachycardia with } \\
\text { poor tolerance }\end{array}$} & ERCP \\
\hline & & & Confusion \\
\hline & & & $\begin{array}{l}\text { Hemoptysis (negative } \\
\text { bronchoscopy) }\end{array}$ \\
\hline & & & COPD exacerbation ( 2 cases) \\
\hline & & \multirow{4}{*}{$\begin{array}{l}\text { Elective admission for } \\
\text { AF ablation } \\
\text { Stroke ( } 2 \text { cases) } \\
\text { AF and heart failure post- } \\
\text { TAVI complicated with IE }\end{array}$} & $\begin{array}{l}\text { Total hip replacement } \\
\text { ( } 2 \text { cases) }\end{array}$ \\
\hline & & & Urinary retention \\
\hline & & & Heat stroke \\
\hline & & & Mixed dementia \\
\hline & & \multicolumn{2}{|l|}{ Elective for CRT-P ( 2 cases) } \\
\hline & & \multicolumn{2}{|l|}{ Ischemic large leg ulcer } \\
\hline & & \multicolumn{2}{|l|}{ Elective admission for EVAR } \\
\hline & & \multicolumn{2}{|l|}{ Pseudoanuerysm } \\
\hline & & \multicolumn{2}{|l|}{ Sternal wall exploration } \\
\hline & & \multicolumn{2}{|l|}{$\begin{array}{l}\text { Worsening gradient } \\
\text { needing re-do TAVI }\end{array}$} \\
\hline & & \multicolumn{2}{|l|}{ Unstable angina } \\
\hline & & \multicolumn{2}{|l|}{$\begin{array}{l}\text { Renal artery stenosis } \\
\text { requiring intervention }\end{array}$} \\
\hline
\end{tabular}

$\mathrm{AF}$ - atrial fibrillation; AVF — arteriovenous fistula; CCF — congestive cardiac failure; CEA — carotid endarterectomy; COPD — chronic obstructive pulmonary disease; CRT — cardiac resynchronization therapy; ERCP - endoscopic retrograde cholangiopancreatography; EVAR - endovascular aneurysm repair; IE - infective endocarditis IV - intravenous; LRTI - lower respiratory tract infection; LAD-PCI - left anterior descending percutaneous coronary intervention; MOF — multi-organ failure; MSK — musculoskeletal; OGD — oesophago-gastro duodenoscopy; PE — pulmonary embolism; PPM — permanent pacemaker; TAVI — transcatheter aortic valve implantation; TIA — transient ischemic attack; UGI - upper gastrointestinal 


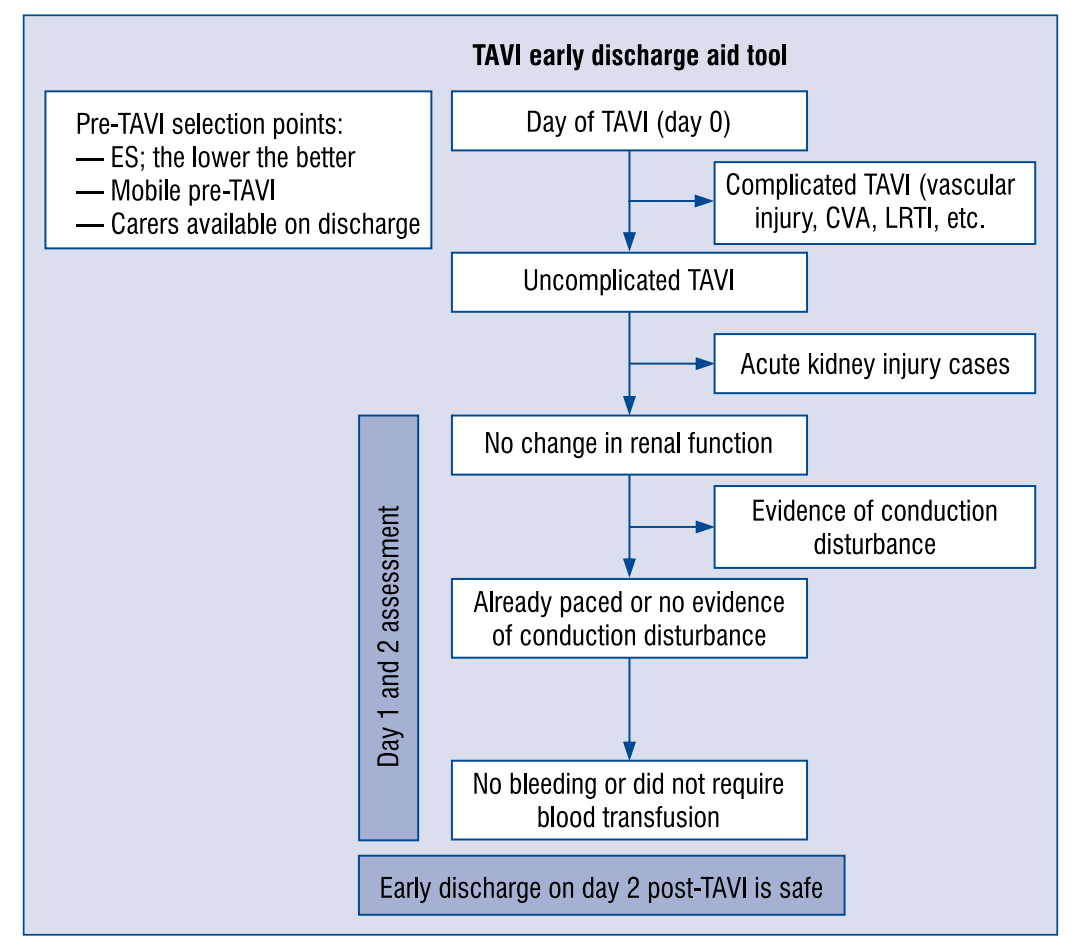

Figure 2. Protocol for post-transcatheter aortic valve implantation (TAVI) early discharge; CVA - cerebrovascular accident; ES - EuroSCORE; LRTI — lower respiratory tract infection.

that this group of patients is heterogenous. Nonetheless, our center has evolved with the technique and offers an experience across these developments in both device technology and practical experience. Finally, the present results may not apply to other prostheses knowing that this was primarily an Edwards Sapien centre.

\section{Conclusions}

Lower logistic EuroSCORE, smaller delta creatinine and not developing any complication were factors associated with early discharge. Moreover, the presented data confirm that early discharge post TAVI is feasible, safe and likely to improve cost-efficiency. Efforts to preserve renal function and avoid bleeding peri-procedurally are likely the two main modifiable predictors of early discharge.

Supporting: National Institute for Health Research Biomedical Research Centre award to Guy's \& St. Thomas' Hospital and King's College London in partnership with King's College Hospital.

Conflict of interest: Omar Aldalati received educational grant from Edwards Lifesciences. Mark Monaghan received research support from Philips and Siemens. Olaf Wendler is a consultant and proctor for Edwards Lifesciences and received research grants and speaker honoraria from Edwards Lifesciences and Medtronic. Philip MacCarthy is a consultant and proctor for Edwards Lifesciences. Other authors have no conflict of interest to declare.

\section{References}

1. Leon MB, Smith CR, Mack MJ, et al. Transcatheter or Surgical Aortic-Valve Replacement in Intermediate-Risk Patients. N Engl J Med. 2016; 374: 1609-1620.

2. Fairbairn TA, Meads DM, Hulme C, et al. The cost-effectiveness of transcatheter aortic valve implantation versus surgical aortic valve replacement in patients with severe aortic stenosis at high operative risk. Heart. 2013; 99(13): 914-920, doi: 10.1136/ heartjnl-2013-303722, indexed in Pubmed: 23696198.

3. Kappetein A, Head S, Généreux P, et al. Updated standardized endpoint definitions for transcatheter aortic valve implantation. J Am Coll Cardiol. 2012; 60(15): 1438-1454, doi: 10.1016/j. jacc.2012.09.001.

4. Vittinghoff E, McCulloch CE. Relaxing the rule of ten events per variable in logistic and Cox regression. Am J Epidemiol. 2007; 165(6): 710-718, doi: 10.1093/aje/kwk052, indexed in Pubmed: 17182981.

5. Durand E, Eltchaninoff H, Canville A, et al. Feasibility and safety of early discharge after transfemoral transcatheter aortic valve implantation with the Edwards SAPIEN-XT prosthesis. Am J Cardiol. 2015; 115(8): 1116-1122, doi: 10.1016/j.amjcard.2015.01.546, indexed in Pubmed: 25726383. 
6. Barbanti M, Capranzano P, Ohno Y, et al. Early discharge after transfemoral transcatheter aortic valve implantation. Heart. 2015; 101(18): 1485-1490, doi: 10.1136/heartjnl-2014-307351, indexed in Pubmed: 26076940.

7. Khawaja MZ, Rajani R, Cook A, et al. Permanent pacemaker insertion after CoreValve transcatheter aortic valve implantation: incidence and contributing factors (the UK CoreValve Collaborative). Circulation. 2011; 123(9): 951-960, doi: 10.1161/CIRCULATIONAHA.109.927152, indexed in Pubmed: 21339482.

8. Franzoni I, Latib A, Maisano F, et al. Comparison of incidence and predictors of left bundle branch block after transcatheter aortic valve implantation using the CoreValve versus the Edwards valve. Am J Cardiol. 2013; 112(4): 554-559, doi: 10.1016/j. amjcard.2013.04.026, indexed in Pubmed: 23726173.

9. Wendler O, Maccarthy P. Renal failure after transcatheter aortic valve implantation: do we know the full story? J Am Coll Cardiol.
2013; 62(10): 878-880, doi: 10.1016/j.jacc.2013.04.058, indexed in Pubmed: 23707322.

10. Yamamoto M, Hayashida K, Mouillet G, et al. Prognostic value of chronic kidney disease after transcatheter aortic valve implantation. J Am Coll Cardiol. 2013; 62(10): 869-877, doi: 10.1016/j. jacc.2013.04.057, indexed in Pubmed: 23707321.

11. Wessely M, Rau S, Lange P, et al. Chronic kidney disease is not associated with a higher risk for mortality or acute kidney injury in transcatheter aortic valve implantation. Nephrol Dial Transplant. 2012; 27(9): 3502-3508, doi: 10.1093/ndt/gfs102.

12. Babaliaros V, Devireddy C, Lerakis S, et al. Comparison of transfemoral transcatheter aortic valve replacement performed in the catheterization laboratory (minimalist approach) versus hybrid operating room (standard approach): outcomes and cost analysis. JACC Cardiovasc Interv. 2014; 7(8): 898-904, doi: 10.1016/j. jcin.2014.04.005, indexed in Pubmed: 25086843. 